\title{
ABORDAGEM DE TEMAS CORRELATOS A ESTOMATERAPIA NO ENSINO DE GRADUAÇÃO EM ENFERMAGEM: ANÁLISE DOCUMENTAL
}

\author{
Francielle Paula de Freitas Morais ${ }^{1, *}\left(\mathbb{D}\right.$, Paulo Henrique Fernandes dos $\operatorname{Santos}^{2}$ (D), \\ Fernanda Letícia Frates Cauduro² (B)
}

\begin{abstract}
RESUMO
Objetivo: discutir a abordagem de temas correlatos à estomaterapia nos Projetos Pedagógicos (PP) e ementas de cursos de graduação de enfermagem de instituições públicas de ensino superior (IPES) do centro-oeste brasileiro. Método: pesquisa documental, exploratória, de abordagem qualitativa, desenvolvida no período de março a junho de 2020. Os cursos de enfermagem foram localizados por meio de acesso ao portal on-line do Ministério da Educação (e-MEC) e acessados os PP e ementas das disciplinas, quando disponíveis. Resultados: compuseram a amostra 17 cursos de graduação em enfermagem. Temas correlatos à estomaterapia estão presentes em disciplinas obrigatórias, optativas ou atividades extensionistas. Identificou-se predominância do conteúdo de feridas em disciplinas obrigatórias, e os temas estomias e incontinências são ofertados principalmente em disciplinas optativas ou atividades extensionistas. Conclusão: a pesquisa almeja ofertar às IPES subsídios para reflexão e avaliação curricular na difusão de temas correlatos à estomaterapia na formação superior em enfermagem.
\end{abstract}

DESCRITORES: Estomia. Enfermagem. Ensino. Educação em enfermagem. Estomaterapia.

\section{ADDRESSING THEMES RELATED TO STOMATHERAPY IN UNDERGRADUATE NURSING EDUCATION: DOCUMENT ANALYSIS}

\begin{abstract}
Objective: discuss the approach of themes related to stomatherapy in Pedagogical Projects (PP) and syllabuses of undergraduate nursing courses from public institutions of higher education (PIHE) in the Brazilian Midwest. Method: documentary research, exploratory, with a qualitative approach, developed from March to June 2020. The nursing courses were located through access to the Ministry of Education's online portal (e-MEC) and the PP and the subject syllabuss were accessed, when available. Results: The sample consisted of 17 graduation nursing courses. Themes related to stomatherapy are present in mandatory, elective or extension activities. It was identified a predominance of wound content in mandatory subjects, and the themes ostomy and incontinence are offered mainly in elective subjects or extension activities. Conclusion: the research aims to offer the PIHE subsidies for reflection and curriculum evaluation in the dissemination of topics related to stomatherapy in higher education in nursing
\end{abstract}

DESCRIPTORS: Ostomy. Nursing. Teaching. Nursing education. Stoma therapy.

1. Escola Superior de Ciências da Saúde do Distrito Federal - Secretaria de Saúde do Distrito Federal - Brasília (DF), Brasil. 2. Universidade de Brasília - Faculdade de Ciências da Saúde - Departamento de Enfermagem - Brasília (DF), Brasil.

*Autora correspondente: franpaula.freitas@gmail.com

Editor de Seção: Isabel Cristina R V Santos

Recebido: Mar. 2021,17 | Aceito: Set. 2021, 21

Como citar: Morais FPF; Santos PHF; Cauduro FLF. Abordagem de temas correlatos a estomaterapia no ensino de graduação em enfermagem: análise documental. ESTIMA, Braz. J. Enterostomal Ther., 2021, 19: e2421. https://doi.org/10.30886/estima. v19.1028_PT 


\title{
ENFOQUE DE TEMAS RELACIONADOS A LA ESTOMATERAPIA EN LA ENSEÑANZA DE GRADUACIÓN EN ENFERMERÍA: ANÁLISIS DOCUMENTAL
}

\begin{abstract}
RESUMEN
Objetivo: discutir el enfoque de temas relacionados a la estomaterapia en los Proyectos Pedagógicos (PP) y descripciones de cursos de graduación de enfermería de instituciones públicas de enseñanza superior (IPES) del centro-oeste brasileño. Método: investigación documental, exploratoria, de enfoque cualitativo, desarrollada en el período de marzo a junio del 2020. Los cursos de enfermería fueron localizados por medio de acceso al portal online del Ministerio de la Educación (e-MEC) y accedidos los PP y descripciones de las asignaturas, cuando estén disponibles. Resultados: compusieron la muestra 17 cursos de graduación en enfermería. Temas relacionados a la estomaterapia están presentes en asignaturas obligatorias, optativas o actividades extensionistas. Se identificó predominancia del contenido de heridas en asignaturas obligatorias, y los temas estomías e incontinencias son ofertados principalmente en asignaturas optativas o actividades extensionistas. Conclusión: la investigación desea ofertar a las IPES subsidios para la reflexión y evaluación curricular en la difusión de temas relacionados a la estomaterapia en la formación superior en enfermería.
\end{abstract}

DESCRIPTORES: Estomía. Enfermería. Enseñanza. Educación en enfermería. Estomaterapia.

\section{INTRODUÇÃO}

Durante a formação universitária, o acadêmico de enfermagem se depara com ampla diversidade de disciplinas e conteúdos. As Diretrizes Curriculares Nacionais para os cursos de graduação em enfermagem orientam para uma formação generalista. Espera-se que o futuro profissional esteja qualificado para atuar e intervir em ações de promoção, prevenção, proteção e reabilitação à saúde, proporcionando integralidade da assistência a indivíduos, famílias e comunidades frente à multiplicidade de problemas e situações de saúde-doença ${ }^{1}$.

Nos três níveis de atenção à saúde, o enfermeiro poderá atender indivíduos com necessidades de saúde que abrangem o campo da estomaterapia, uma especialidade da enfermagem que visa o cuidado de indivíduos com estomias, feridas agudas e crônicas, fístulas, tubos, cateteres, drenos, incontinência anal e urinária².

Como especialidade, a estomaterapia surgiu no final da década de 50 nos Estados Unidos, mas somente em 1980 foi incluída como especialidade para a enfermagem pelo World Council of Enterostomal Therapists (WCET). Essa entidade visa garantir que o atendimento especializado de enfermagem esteja disponível em todo o mundo para as pessoas necessitadas de cuidados de estomia, feridas ou continência ${ }^{3}$. No Brasil, o marco da estomaterapia ocorreu a partir da realização do primeiro curso de especialização oferecido pela Escola de Enfermagem da Universidade de São Paulo em $1990^{2}$.

A procura por essa especialidade é crescente e um estudo conduzido com enfermeiros estomaterapeutas egressos de um curso de especialização no Brasil revelou que, na população estudada, 70\% atuavam na área, com predominância para o cuidado de estomias e feridas ${ }^{4}$.Embora os dados revelem elevada inserção desses profissionais no mercado de trabalho, considera-se premente pontuar que a assistência de enfermagem a pacientes com demandas desse campo também é ofertada por enfermeiros generalistas.

Nesse aspecto, enfermeiros não estomaterapeutas, mas que assistem indivíduos com tais morbidades, muitas de alta complexidade, devem estar preparados para prestar os cuidados mínimos e, quando couber, identificar a necessidade de encaminhamento para serviço especializado ${ }^{5}$. Considera-se, portanto, que durante a graduação em enfermagem é fundamental a oferta de conteúdos que envolvam a estomaterapia, a fim de que o egresso possa desenvolver competências mínimas para atender esses pacientes.

Mediante a observação dos pesquisadores durante a realização de atividades didático- pedagógicas em uma instituição pública de ensino superior (IPES) do centro-oeste brasileiro, e percepção de fragilidades no conhecimento de acadêmicos de enfermagem sobre conteúdos relacionados a feridas, estomias e incontinência, questionou-se como as instituições de ensino têm contemplado conteúdo do escopo da estomaterapia na formação de enfermeiros. 
Esta pesquisa teve por objetivo discutir a abordagem de temas correlatos à estomaterapia nos Projetos Pedagógicos (PP) e ementas de cursos de graduação de enfermagem das IPES do centro-oeste brasileiro. Espera-se que os dados produzidos contribuam na formação de enfermagem a nível superior ao provocar a reflexão de docentes sobre a pertinência da inclusão, ou ampliação, de conteúdos associados à estomaterapia para geração de impacto positivo na assistência, bem como fornecer elementos que apoiem o desenvolvimento de pesquisas de base curricular.

\section{MÉTODO}

Trata-se de uma pesquisa documental, exploratória, de abordagem qualitativa, desenvolvida no período de março a junho de 2020, cuja questão de pesquisa foi "Como ocorre a abordagem de temas correlatos à estomaterapia nos cursos de graduação em enfermagem das IPES do centro-oeste do Brasil?”. Para a obtenção de dados foram seguidas as etapas descritas por Rodrigues ${ }^{6}$ : identificação, seleção e elegibilidade. A etapa de identificação foi realizada a partir do acesso ao portal de instituições de educação superior do Ministério da Educação (e-MEC). Na segunda etapa, de seleção, identificaram-se os cursos de graduação em enfermagem presenciais de IPES, habilitações bacharelado e licenciatura. A elegibilidade ocorreu por meio do acesso às páginas on-line dos cursos e verificada a disponibilidade do PP e ementa das disciplinas, sendo incluídas as instituições que dispunham de tais informações.

Ao serem exploradas as páginas na web, notaram-se indicativos do desenvolvimento, em algumas instituições, de atividades de extensão com foco na estomaterapia. Desse modo, essas informações também foram compiladas.

Todos os PP e ementas foram lidos na íntegra. Para identificar conteúdos correlatos à estomaterapia, buscaram-se os

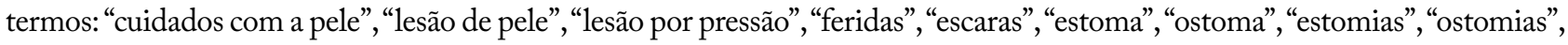
“estomizados”, "ostomizados", “incontinência(s)”, "fístulas", “drenos”, "cateteres”, "tubos” e "estomaterapia”. Ressalta-se que foram consideradas todas as derivações e prefixos associados ao termo "estomias". Foram excluídas informações que continham o termo "pele" contidas nas disciplinas básicas do currículo (a exemplo: anatomia e fisiologia). O mesmo critério foi aplicado para termos identificados no descritivo e especificação de insumos para treino de habilidades em laboratórios de enfermagem.

A tabulação das informações foi realizada em planilha do Excel $^{\circledR}$ com o registro dos elementos: Estado, IPES, carga horária do curso, ano do PP, número total de disciplinas por semestre, indicação do semestre do curso e atividade de extensão. $\mathrm{Na}$ identificação das IPES foram aplicados códigos de acordo com o Estado da Federação e números consecutivos para fins de organização. Para a interpretação, utilizou-se a análise de conteúdo temático-categorial, baseado nos pressupostos da análise de conteúdo de Bardin, sendo elas: leitura exaustiva dos PP e ementas, construção de hipóteses provisórias acerca do objeto de estudo, determinação das unidades de registro, definição das unidades de significação e quantificação dos temas, agrupamento dos temas a partir de critérios teóricos e apresentação dos resultados 7 .

Por se tratar de dados de domínio público, o projeto não foi submetido ao Comitê de Ética em Pesquisa, seguindo as orientações do Conselho Nacional da Saúde no 510/2016.

\section{RESULTADOS}

Foram identificadas no sistema e-MEC 19 IPES do centro-oeste do Brasil, entretanto 2 instituições não atenderam aos critérios de inclusão pré-estabelecidos. Desse modo, a análise final foi realizada nos documentos de 17 cursos de graduação em enfermagem, distribuídos nos estados de Goiás ( $n=05)$, Mato Grosso ( $n=05)$, Mato Grosso do Sul ( $n=04)$ e no Distrito Federal $(n=03)$.

Com relação à abordagem de temas correlatos à estomaterapia, o corpus submetido à análise emergiu de disciplinas obrigatórias, optativas e atividades de extensão.

Após leitura flutuante e intuitiva dos dados e definição das hipóteses provisórias7, organizaram-se as unidades de registro, temas e categorias. Nesse aspecto, foi possível estabelecer a abordagem de temas correlatos à estomaterapia sob três categorias temáticas: Abordagem em disciplinas obrigatórias; Abordagem em disciplinas optativas; Abordagem em atividades de extensão. 


\section{Categoria 1: Abordagem de temas correlatos à estomaterapia em disciplinas obrigatórias}

Entre os PP e ementas analisados, em 16 foi localizado pelo menos 1 termo. Esses estão inseridos na descrição de ementas, conteúdos, tópicos de ensino ou referências bibliográficas, de consulta obrigatória ou complementar. Com isso, nesta categoria definiram-se duas subcategorias: A estomaterapia em ementas, conteúdos e tópicos de ensino e Bibliografia para ensino de temas correlatos à estomaterapia.

\section{A estomaterapia em ementas, conteúdos e tópicos de ensino}

Os termos que mais se repetiram nessa subcategoria foram "feridas", "curativos" e "integridade da pele"; em apenas uma ementa localizou-se o termo "cateterismo", "drenos" e o sufixo "ostomia". Verificou-se que o estudante de graduação em enfermagem aproxima-se do tema entre o terceiro e sexto semestre do curso, em disciplinas com foco no conhecimento e treino de habilidades (fundamentos de enfermagem, semiotécnica/semiologia e processo de cuidar). A apresentação dos resultados encontra-se na Tabela 1.

Tabela 1. Distribuição dos dados das instituições públicas de ensino superior de acordo com o semestre, disciplina, ementa, conteúdo e tópico de ensino. Brasília (DF) - 2020.

\begin{tabular}{|c|c|c|c|}
\hline IPES & Semestre & Disciplina & Ementa/ Conteúdo/ Tópico de ensino \\
\hline Enf/DF1 & $4^{\circ}$ semestre & $\begin{array}{l}\text { Semiologia e } \\
\text { Semiotécnica }\end{array}$ & $\begin{array}{l}\text { Assistência de Enfermagem empregada no déficit tegumentar: cuidado com } \\
\text { feridas; cuidado com drenos. Assistência de Enfermagem empregada no déficit } \\
\text { nutricional e hidroeletrolítico: cateterismo gástrico; cateterismo duodenal; } \\
\text { cateterismo vesical intermitente e de demora. Assistência de Enfermagem } \\
\text { empregada no déficit ventilatório: traqueostomia. }\end{array}$ \\
\hline Enf/GO1 & $6^{\circ}$ semestre & $\begin{array}{l}\text { Enfermagem } \\
\text { Cirúrgica }\end{array}$ & Medidas profiláticas relacionadas às infecções de feridas cirúrgicas. \\
\hline Enf/GO2 & $5^{\circ}$ semestre & $\begin{array}{l}\text { Semiologia e } \\
\text { Semiotécnica II }\end{array}$ & Curativos: técnicas e coberturas. Novas tecnologias terapêuticas. \\
\hline Enf/GO3 & $5^{\circ}$ semestre & $\begin{array}{l}\text { Semiologia e } \\
\text { Semiotécnica II }\end{array}$ & Curativos: técnicas c coberturas. \\
\hline Enf/MT2 & $4^{\circ}$ semestre & $\begin{array}{l}\text { Processo de } \\
\text { Cuidar I }\end{array}$ & $\begin{array}{l}\text { Ênfase nas necessidades humanas básicas de Oxigenação, Circulação, } \\
\text { Termorregulação, Higiene, Conforto e Integridade da pele. }\end{array}$ \\
\hline Enf/MT3 & $4^{\circ}$ semestre & $\begin{array}{l}\text { Processo de } \\
\text { Cuidar I }\end{array}$ & $\begin{array}{l}\text { Ênfase nas necessidades humanas básicas de Oxigenação, Circulação, } \\
\text { Termorregulação, Higiene, Conforto e Integridade da pele. }\end{array}$ \\
\hline Enf/MT4 & $4^{\circ}$ semestre & $\begin{array}{l}\text { Processo de } \\
\text { Cuidar I }\end{array}$ & $\begin{array}{l}\text { Ênfase nas necessidades humanas básicas de Oxigenação, Circulação, } \\
\text { Termorregulação, Higiene, Conforto e Integridade da pele. }\end{array}$ \\
\hline Enf/MS1 & $\begin{array}{l}3^{\circ} \text { e } 4^{\circ} \\
\text { semestre }\end{array}$ & $\begin{array}{l}\text { Fundamentos de } \\
\text { Enfermagem }\end{array}$ & Tratamento de feridas. \\
\hline Enf/MS3 & $3^{\circ}$ semestre & $\begin{array}{l}\text { Fundamentos de } \\
\text { Enfermagem I }\end{array}$ & $\begin{array}{l}\text { Assistência de enfermagem frente às necessidades humanas básicas } \\
\text { relacionadas à higiene, conforto e oxigenação; comunicação e registros de } \\
\text { enfermagem; manutenção das funções reguladoras; posicionamento do paciente; } \\
\text { terminologia científica de enfermagem; manutenção da integridade corporal e } \\
\text { cuidados com feridas. }\end{array}$ \\
\hline Enf/MS4 & $3^{\circ}$ semestre & $\begin{array}{l}\text { Fundamentos de } \\
\text { Enfermagem I }\end{array}$ & $\begin{array}{l}\text { Assistência de enfermagem frente às necessidades humanas básicas } \\
\text { relacionadas à higiene e conforto, manutenção da integridade corporal e } \\
\text { cuidados com feridas. }\end{array}$ \\
\hline
\end{tabular}

IPES= Instituição Pública de Ensino Superior; Enf= Enfermagem; DF= Distrito Federal; GO= Goiás; MT= Mato Grosso; MS= Mato Grosso do Sul. 


\section{Bibliografia para ensino de temas correlatos à estomaterapia}

Identificaram-se oito referências bibliográficas distribuídas nas ementas de sete IPES (Tabela 2). Dessas, em apenas uma foi observada a associação entre disciplina, descrição de tema/conteúdo e bibliografia com abordagem de tema correlato à estomaterapia.

Com relação à característica das referências, foi unânime o uso de livros com enfoque no cuidado geral de feridas $(n=07)$ e 1 específica para cuidados com lesões por pressão. Não foram identificadas referências obrigatórias sobre estomias ou incontinências. O ano de publicação dos livros variou entre 2007 e 2014, todos de produção nacional.

Tabela 2. Distribuição dos dados das instituições públicas de ensino superior de acordo com o semestre, disciplina e referência bibliográfica obrigatória e complementar. Brasília (DF) - 2020.

\begin{tabular}{|c|c|c|c|}
\hline IPES & Semestre(s) & Disciplina(s) & Referência bibliográfica - obrigatória \\
\hline $\begin{array}{l}\text { Enf/DF2 } \\
\text { Enf/GO4 } \\
\text { Enf/MS2 }\end{array}$ & $\begin{array}{l}4^{\circ} \text { semestre } \\
4^{\circ} \text { semestre } \\
7^{\circ} \text { semestre }\end{array}$ & $\begin{array}{l}\text { Fundamentos de Enfermagem. } \\
\text { Bases para o cuidar do Indivíduo e da Família II. } \\
\text { Adulto e Idoso. } \\
\text { Dimensões do Cuidar em Enfermagem IV. }\end{array}$ & $\begin{array}{l}\text { Borges EL, et al. Feridas: Como Tratar. } 1^{\circ} \text { ed. Belo } \\
\text { Horizonte: Coopmed; 2007. } 248 \text { p } \\
\text { Dealey C. Cuidando de feridas: um guia para as } \\
\text { enfermeiras. } 3^{\text {a }} \text { ed. São Paulo: Atheneu Editora; } \\
\text { 2008. 248p. }\end{array}$ \\
\hline Enf/DF2 & $6^{\circ}$ semestre & Vivências Integradoras 5 & $\begin{array}{l}\text { Blanes L, Ferreira LM. Prevenção e tratamento de } \\
\text { úlcera por pressão. São Paulo: Atheneu; } 2014 .\end{array}$ \\
\hline $\begin{array}{l}\text { Enf/DF2 } \\
\text { Enf/MS1 }\end{array}$ & $\begin{array}{l}6^{\circ} \text { semestre } \\
3^{\circ}, 4^{\circ}, 5^{\circ} \mathrm{e} \\
6^{\circ} \text { semestres }\end{array}$ & $\begin{array}{l}\text { Fundamentos de Enfermagem II } \\
\text { Enfermagem em Saúde do Adulto e do Idoso I }\end{array}$ & $\begin{array}{l}\text { Jorge AS, Dantas SRPE. Abordagem } \\
\text { multiprofissional de ferida. Campinas: Atheneu, } \\
2003 .\end{array}$ \\
\hline Enf/GO4 & $4^{\circ}$ semestre & Bases para o cuidar do Indivíduo e da Família II & 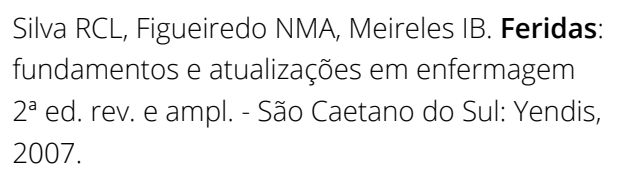 \\
\hline Enf/MT1 & $4^{\circ}$ semestre & O processo de cuidar: fundamentação e prática. & 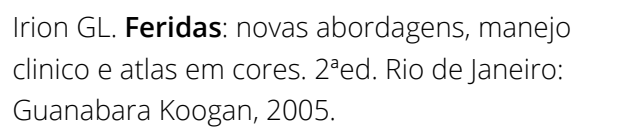 \\
\hline Enf/MT4 & $\begin{array}{l}4^{\circ} \text { semestre } \\
9^{\circ} \text { e } 10^{\circ} \\
\text { semestre }\end{array}$ & $\begin{array}{l}\text { Processo de Cuidar I. } \\
\text { Estágio Supervisionado I e II }\end{array}$ & $\begin{array}{l}\text { Blanck M, Giannini T. Úlceras e feridas - As } \\
\text { feridas têm alma. Di livros, } 2014 . \\
\text { Geovanini T. Tratado de Feridas e Curativos: } \\
\text { Enfoque Multiprofissional. Bahia: Rideel, } 2013 . \\
\text { Malagutti W. Feridas: conceitos e atualidades. } \\
1^{\text {a }} \text { ed. Martinari: } 2015 .\end{array}$ \\
\hline Enf/DF3 & $\begin{array}{l}7^{\circ} \text { e } 8^{\circ} \\
\text { semestres }\end{array}$ & $\begin{array}{l}\text { Estágio Curricular Obrigatório em Atenção } \\
\text { Hospitalar (Saúde da Mulher/Recém-Nascido, } \\
\text { Criança/Adolescente, Adulto/ Idoso) }\end{array}$ & $\begin{array}{l}\text { Blanes L, Ferreira LM. Prevenção e tratamento de } \\
\text { úlcera por pressão. São Paulo: Atheneu; } 2014 .\end{array}$ \\
\hline
\end{tabular}

IPES= Instituição Pública de Ensino Superior; Enf= Enfermagem; DF= Distrito Federal; GO= Goiás; MT= Mato Grosso; MS= Mato Grosso do Sul.

\section{Categoria 2: Abordagem de temas correlatos à estomaterapia em disciplinas optativas}

Entre as IPES, duas ofertavam disciplinas optativas voltadas ao cuidado e tratamento de feridas, duas à assistência de enfermagem em estomaterapia, uma aos cuidados de "pessoas com estomia" e uma incluía a prevenção de lesões por pressão como tópico da gestão de riscos e segurança do paciente (Tabela 3). 
Tabela 3. Distribuição dos dados das instituições públicas de ensino superior de acordo com disciplina e ementa. Brasília (DF) - 2020.

\begin{tabular}{|c|c|c|}
\hline IPES & Disciplina & Ementa \\
\hline Enf/DF2 & $\begin{array}{l}\text { Cuidados de Enfermagem em Feridas } \\
\text { Agudas e Crônicas }\end{array}$ & $\begin{array}{l}\text { Disciplina teórico-prática que desenvolve as bases conceituais da } \\
\text { assistência de enfermagem a clientes com feridas crônicas e agudas, com } \\
\text { enfoque na prevenção e no tratamento de feridas. }\end{array}$ \\
\hline Enf/GO1 & $\begin{array}{l}\text { A segurança do paciente no ambiente } \\
\text { hospitalar }\end{array}$ & $\begin{array}{l}\text { A segurança para o paciente de acordo com a legislação vigente: } \\
\text { protocolos de prevenção de quedas, segurança de prescrição, } \\
\text { identificação do paciente, prática de higiene das mãos, cirurgias seguras e } \\
\text { prevenção de úlceras por pressão. }\end{array}$ \\
\hline Enf/GO2 & Enfermagem em Estomaterapia & $\begin{array}{l}\text { Estomas, incontinência anal, urinárias e feridas. Cuidados e materiais } \\
\text { específicos utilizados na assistência. Ciência de conhecimentos técnicos e } \\
\text { princípios da relação de ajuda e autonomia. }\end{array}$ \\
\hline Enf/GO3 & Assistência em Estomaterapia & Não disponível \\
\hline Enf/MT5 & $\begin{array}{l}\text { Cuidados de Enfermagem em Pessoas } \\
\text { Ostomizadas }\end{array}$ & $\begin{array}{l}\text { Apresentar equipamentos e adjuvantes utilizados no cuidado de estomas } \\
\text { intestinais, além de discutir os aspectos nutricionais, psicossociais e de } \\
\text { sexualidade dos pacientes submetidos a estomas intestinais. Discutir } \\
\text { aspectos da reabilitação dos pacientes com estomas intestinais, políticas } \\
\text { de inclusão e direitos humanos. }\end{array}$ \\
\hline Enf/MT5 & $\begin{array}{l}\text { Assistência de Enfermagem ao Paciente } \\
\text { com Feridas }\end{array}$ & $\begin{array}{l}\text { Anatomia e fisiologia da pele - evolução da cicatrização - fatores que } \\
\text { interferem no tratamento de feridas - princípios gerais para o tratamento } \\
\text { de feridas - etiologia das principais úlceras - limpeza e debridamento de } \\
\text { feridas - mitos e verdades - coberturas e novas abordagens e alternativas } \\
\text { para o tratamento de feridas - protocolos de tratamento de feridas. }\end{array}$ \\
\hline
\end{tabular}

IPES= Instituição Pública de Ensino Superior; Enf= Enfermagem; DF= Distrito Federal; GO= Goiás; MT= Mato Grosso; MS= Mato Grosso do Sul.

Dentre as IPES que ofertavam disciplinas optativas, apenas duas citavam as referências obrigatórias básicas e complementares (Tabela 4).

Tabela 4. Distribuição dos dados das instituições públicas de ensino superior de acordo com a referência bibliográfica básica e complementar para disciplinas optativas. Brasília (DF) - 2020.

\begin{tabular}{cl}
\hline IPES & Referências Básicas \\
\hline \multirow{2}{*}{ Enf/DF2 } & $\begin{array}{l}\text { Borges EL, et al. Feridas: Como Tratar. 1ed. Belo Horizonte: Coopmed; 2007. } 248 \text { p } \\
\text { Blanes L, Ferreira LM. Prevenção e tratamento de úlcera por pressão. São Paulo: Atheneu; } 2014 . \\
\\
\text { Yamada BFA. Pele - manto protetor: higiene e hidratação. São Paulo: Andreoli, } 2015 .\end{array}$ \\
\hline & $\begin{array}{l}\text { Santos VLCG, Cesaretti IUR. Assistência em estomaterapia: cuidando do ostomizado. São Paulo: Atheneu; 2000. 532p. } \\
\text { Carvalheira C. Ainda posso levar uma vida normal? 2a . ed. Rio de Janeiro: News Eventos \& Promoções; 1999. (Ministério } \\
\text { da Justiça Secretaria de Estado dos Direitos Humanos). } \\
\text { Paula MAB de, Paula PR de, Cesaretti IUR. Estomaterapia: temas básicos em estomas. São Paulo: Cabral; 2006. }\end{array}$ \\
\hline Enf/DF2 & $\begin{array}{l}\text { Domansky RC, Borges EL. Manual para prevenção de lesões de pele: recomendações baseadas em evidências. Rubio. } \\
\text { Dealey C. Cuidando de feridas: um guia para as enfermeiras. 3a ed. São Paulo. Atheneu Editora, 2008. 248p. }\end{array}$ \\
\hline
\end{tabular}

IPES= Instituição Pública de Ensino Superior; Enf= Enfermagem; DF= Distrito Federal; GO= Goiás.

\section{Categoria 3: Abordagem de temas correlatos à estomaterapia em atividades de extensão}

Em quatro páginas na web dos cursos de enfermagem consultados foram notadas atividades extensionistas (projetos de extensão, liga acadêmica e serviço à comunidade) vinculados à estomaterapia, porém em apenas duas a descrição das 
atividades estava disponível (Tabela 5). Destaca-se como tema central de trabalho a avaliação e tratamento de feridas (agudas e crônicas) e a uropediatria.

Tabela 5. Distribuição dos dados das instituições públicas de ensino superior de acordo com a modalidade da atividade de extensão. Brasília (DF) - 2020.

\begin{tabular}{|c|c|c|}
\hline IPES & Modalidade da atividade de extensão & Descrição das atividades de extensão \\
\hline Enf/DF1 & $\begin{array}{l}\text { Liga Acadêmica de Feridas } \\
\text { em Enfermagem }\end{array}$ & $\begin{array}{l}\text { A Liga Acadêmica de Feridas em Enfermagem pretende congregar alunos } \\
\text { do curso de Enfermagem da mesma unidade, visando integrá-los aos } \\
\text { cenários da prática profissional. Tem como princípios o conhecimento, a } \\
\text { educação e a assistência, como as inúmeras ligas acadêmicas criadas em } \\
\text { outras universidades do Brasil e do mundo. }\end{array}$ \\
\hline \multirow{2}{*}{ Enf/DF2 } & $\begin{array}{l}\text { Projeto: Prática avançada de } \\
\text { Enfermagem em Uropediatria }\end{array}$ & Não disponível \\
\hline & $\begin{array}{l}\text { Serviço Ambulatorial de Enfermagem } \\
\text { em estomaterapia }\end{array}$ & Não disponível \\
\hline Enf/GO3 & $\begin{array}{l}\text { Projeto: Prevenção de úlceras por } \\
\text { pressão em idosos do município de } \\
\text { Itumbiara-GO }\end{array}$ & Não disponível \\
\hline Enf/ MT5 & $\begin{array}{l}\text { Projeto: Grupo de Estudos em } \\
\text { Feridas Crônicas }\end{array}$ & $\begin{array}{l}\text { Objetivo: oportunizar momentos de estudo, discussão e prática } \\
\text { sobre avaliação e tratamento de feridas crônicas entre acadêmicos } \\
\text { e docentes do curso de enfermagem como forma de aprimorar os } \\
\text { conhecimentos dos participantes acerca dos cuidados de enfermagem no } \\
\text { tratamento de feridas (....). }\end{array}$ \\
\hline
\end{tabular}

IPES= Instituição Pública de Ensino Superior; Enf= Enfermagem; DF= Distrito Federal; GO= Goiás; MT= Mato Grosso.

\section{DISCUSSÃO}

A presente pesquisa documental possibilitou explorar entre as IPES do centro-oeste brasileiro como os temas correlatos à estomaterapia são abordados nos $\mathrm{PP}$ e ementas das disciplinas dos cursos de graduação em enfermagem. Esses estão presentes em disciplinas obrigatórias, optativas ou ações extensionistas propostas nos cursos.

Cabe enfatizar que o ensino de enfermagem prevê a formação de profissionais críticos e reflexivos, aptos para o trabalho em saúde e atenção aos indivíduos, famílias e comunidades em seus diferentes ciclos de vida, segundo as suas necessidades de saúde ${ }^{1}$. Assistir pacientes com demandas de saúde inclusas no campo da estomaterapia é uma competência a ser desenvolvida durante a graduação, haja vista a necessidade de assistência qualificada aos doentes crônicos, grupo esse com maior prevalência de lesões de pele, estomias, incontinências, ou que fazem uso de cateteres ou drenos ${ }^{5}$.

Em relação às disciplinas obrigatórias, os resultados obtidos são semelhantes ao de estudo realizado no Rio de Janeiro em uma IPES. Os temas correlatos à estomaterapia foram predominantemente relacionados a feridas, cujo ensino é iniciado a partir do terceiro semestre do curso e se estende até o último semestre com conteúdos sobre o processo de cicatrização, classificação e os principais tipos de coberturas utilizadas na prevenção e tratamento das lesões 5 .

Assistir pacientes com feridas faz parte do processo assistencial do enfermeiro, sendo esse profissional responsável por avaliar, prescrever e executar curativos bem como supervisionar a equipe de enfermagem ${ }^{8}$. É essencial, portanto, que no espaço acadêmico sejam ofertados os subsídios para a construção de tais competências para a garantia de uma assistência segura.

$\mathrm{Na}$ graduação em enfermagem é crescente o uso de metodologias inovadoras e emancipatórias no ensino de temas do campo da estomaterapia. Essas têm potencial de reforçar as habilidades cognitivas, permitir o treino de habilidades e a vivência de situações semelhantes às que encontrarão na prática clínica. A simulação clínica de alta fidelidade, realizada com pacientes simulados, role play e moulage, favorecem o raciocínio clínico e a tomada de decisão na avaliação e tratamento de 
feridas ${ }^{9,10}$. Jogos educativos e uso dos princípios da gamificação também são reportados como estratégias aplicáveis para o ensino de conteúdos relacionados às estomias e aos curativos ${ }^{11,12}$.

Com os dados compilados nessa pesquisa, infere-se que os anos iniciais do curso de enfermagem são a base para o conhecimento e aprendizado das habilidades mínimas para o cuidado de feridas, os quais são resgatados nos anos subsequentes por meio de suporte teórico em referências bibliográficas básicas e/ou complementares.

Nas disciplinas obrigatórias, os temas estomia, incontinência, cateteres e drenos não foram identificados, ou apareceram em menor frequência. Esse dado é corroborado por estudo análogo, que sinaliza a necessidade de introduzir o assunto já na graduação, diante da prevalência de problemas de incontinência, principalmente em idosos, mulheres, crianças, e do sofrimento psicofisiológico do acometido por tal agravo, que influencia diretamente na qualidade de vida 5 .

No que se refere às referências bibliográficas, entre as identificadas, duas são específicas sobre lesão por pressão (LP). Esse conteúdo é relevante para a formação profissional, haja vista que em 2013 foi instituído pelo Ministério da Saúde o Plano Nacional de Segurança do Paciente (PNSP), e com ele divulgadas as metas internacionais de segurança do paciente, uma delas direcionada à prevenção de LP nos serviços de saúde.

Segundo o relatório nacional de incidentes relacionados à assistência à saúde, no período de junho de 2019 a maio de 2020, dos 151.442 incidentes notificados, 29.416 (19,42\%) corresponderam às LP, sendo, nesse período, o segundo tipo de evento adverso mais notificado pelos serviços de saúde do país ${ }^{13}$. Destarte, devido à necessidade de prevenir, identificar e tratar as LP, é emergente a inclusão desse tema na formação do enfermeiro.

Nas IPES analisadas, os livros são a fonte primária para a teorização dos conteúdos. Tal fato é associado ao acervo bibliotecário das instituições que, na sua maioria, dispõe de literaturas básicas e tradicionais para a formação profissional. Os livros identificados na pesquisa são exclusivamente produções nacionais dos últimos 10 anos.

Pondera-se que é necessária a busca por referenciais atualizados e que cabe ao docente e instituição de ensino incentivar e disponibilizar aos estudantes o acesso a periódicos e publicações sobre as novidades no campo da estomaterapia. Nesse aspecto, projeta-se a pertinência da difusão da prática baseada em evidências e a inserção curricular dessa proposta para que permeie todo o processo de formação profissiona $1^{14}$. Sugere-se a inclusão nas referências de protocolos clínicos, ensaios clínicos randomizados e as revisões sistemáticas da literatura.

Entre os resultados oportunizados pela pesquisa está a detecção de terminologias obsoletas como úlcera por pressão e ostomias. Em 2016, o National Pressure Ulcer Advisory Panel (NPUAP), organização dedicada à prevenção e ao tratamento de LP, anunciou a mudança do termo "úlcera por pressão" por "lesão por pressão", por descrever com mais precisão as lesões em peles intactas e ulceradas ${ }^{15}$. Desde 2004 o termo "ostomia" foi modificado para "estomia", após consulta da Sociedade Brasileira de Estomaterapia à Academia de Brasileira de Letras $^{16}$.

No que diz respeito às disciplinas optativas, foi identificada a oferta por seis IPES distribuídas entre os Estados de Goiás, Mato Grosso e o Distrito Federal. Essas disciplinas configuram alternativas para promover melhor compreensão dos temas: avaliação, prevenção e tratamento de feridas (agudas e crônicas), atenção aos pacientes com estomia, incontinências urinária e anal e prevenção do evento adverso LP.

Essa modalidade de disciplinas compõe o currículo, mas o acadêmico é autônomo para optar ou não por sua realização. De modo geral, as disciplinas optativas visam propiciar formação mais personalizada em determinado tema ou assunto ${ }^{1}$. Com o crescente número de pacientes com lesões de pele, estomias e incontinência urinária ou anal, além do surgimento de equipamentos e tecnologias no tratamento desses problemas de saúde ${ }^{17}$, defende-se a inclusão nos currículos de graduação em enfermagem de disciplinas que permitam os acadêmicos expandirem suas competências, preparando-os para a assistência desses pacientes.

Reitera-se essa afirmação ao se notar na literatura estudos que indicam fragilidades no conhecimento dos acadêmicos de enfermagem nos conteúdos de feridas ${ }^{18} \mathrm{e} \mathrm{LP}^{19}$. As incontinências foram citadas em apenas uma disciplina que está inclusa no rol das optativas. Estudo que identificou baixo conhecimento do assunto "incontinência urinária" entre acadêmicos de enfermagem, alerta para a necessidade da revisão dos programas de aprendizagem com vistas à ampliação de sua abordagem ${ }^{20}$. Em relação às estomias, criar oportunidades para que o acadêmico treine habilidades para o esvaziamento, seleção de dispositivos e troca da bolsa de colostomia pode elevar a confiança para esse tipo de cuidado ${ }^{21}$. 
O suporte à formação do enfermeiro também perpassa pelo envolvimento com atividades extensionistas. Os achados da pesquisa indicaram cinco atividades de extensão voltadas à atenção a pacientes com feridas crônicas, agudas e incontinências. Dessas, três estão concentradas no Distrito Federal: uma Liga acadêmica e dois projetos de extensão.

As atividades extensionistas devem ser parte integrante do currículo de graduação das instituições de ensino superior. Essas preveem a interação dialógica entre ambiente acadêmico e comunidade, a fim de propiciar a troca de conhecimentos e o trabalho interdisciplinar e interprofissional. De modo mais recente, tem-se ampliado esse debate em face da inserção curricular da extensão em que é previsto a inclusão de ações extensionistas em no mínimo $10 \%$ da carga horária curricular estudantil ${ }^{22}$.

Nesse sentido, o vínculo entre academia e comunidade é referenciado nas descrições compiladas em duas atividades de extensão. Oferecer serviços para além dos muros da Universidade é um compromisso das IPES. Nesse processo, além de proporcionar aprendizado sobre a especialidade aos enfermeiros em formação ${ }^{23}$, colabora-se com a rede de atenção à saúde ao se prover mais um ponto de assistência à população.

Uma experiência de Liga Acadêmica em Estomaterapia no Brasil relata o aprimoramento dos acadêmicos participantes frente aos temas feridas, estomias e incontinências, a relevância da experiência adquirida na extensão e aplicabilidade dos conteúdos estudados para o desempenho da prática profissional ${ }^{23}$.

Aponta-se como limitação do estudo a ausência dos documentos disponíveis on-line e descrição minuciosa das atividades de extensão desenvolvidas no âmbito dos cursos de enfermagem analisados. Ademais, foram avaliados apenas documentos públicos, disponíveis na página oficial dos cursos de graduação consultados, sendo pertinente observar programas de aprendizagem, planos de disciplinas e o currículo oculto.

Frente ao exposto e com vistas à intensificação na abordagem de conteúdos da estomaterapia durante a graduação em enfermagem, sugere-se olhar atento das instituições para a relevância dos temas para a prática profissional. Cabe ainda o incentivo aos acadêmicos sobre a busca por atualizações que fundamentem sua prática clínica ${ }^{24} \mathrm{e}$ os preparem para cuidar de indivíduos com feridas, incontinências e estomias.

\section{CONCLUSÃO}

Ao analisar os PP e ementas dos cursos de graduação de enfermagem das IPES do centro-oeste brasileiro, foi possível identificar predominância do conteúdo feridas e, em menor proporção, estomias e incontinências. $\mathrm{O}$ assunto é abordado a partir do terceiro semestre, em disciplinas obrigatórias, com foco no conhecimento e treino de habilidades, apoiado em referenciais bibliográficos dos últimos 10 anos.

Em se tratando de estomias e incontinências, conclui-se que é oportunizado ao acadêmico a ampliação do conhecimento nessas temáticas em disciplinas optativas ou atividades extensionistas. Nesse aspecto, participarão dessas atividades acadêmicos que tenham interesse em se aprofundar nesses assuntos, o que não exclui a necessidade de ofertá-los em disciplinas do fluxo obrigatório.

Considera-se necessário que egressos de enfermagem tenham conhecimento mínimo sobre o conceito de estomias, os diferentes tipos, indicações, complicações, cuidados gerais de enfermagem e habilidades para encaminhar os pacientes com necessidade de cuidado especializado.

Em relação à temática incontinência, a graduação tem o papel de socializar conhecimentos e suscitar reflexões aos graduandos sobre a importância do profissional enfermeiro no atendimento aos pacientes acometidos pelos diversos tipos de incontinência.

Entende-se que o objetivo das instituições é a formação generalista, porém é notória a necessidade da apreensão de conhecimentos e desenvolvimento de habilidades específicas que são requeridas na prática profissional.

A presente pesquisa almeja agregar valor para o ensino superior de enfermagem, ofertando às IPES e demais instituições subsídios para reflexão e avaliação curricular na difusão de temas correlatos à estomaterapia na formação superior em enfermagem.

Para aprofundar as investigações sobre o ensino da estomaterapia, sugere-se que estudos mais abrangentes e que incluam programas de aprendizagem, planos de ensino e o currículo oculto, a fim de que seja possível obter o panorama sobre o ensino de temas correlatos à estomaterapia na formação do enfermeiro a âmbito nacional. 


\section{CONTRIBUIÇÃO DOS AUTORES}

Conceitualização: Morais FPF e Cauduro FLF; Metodologia: Morais FPF e Cauduro FLF; Investigação: Morais FPF; Redação - Primeira versão: Morais FPF, Santos PHF e Cauduro FLF; Redação-Revisão \& Edição: Morais FPF, Santos PHF e Cauduro FLF; Supervisão: Cauduro FLF.

\section{DISPONIBILIDADE DE DADOS DE PESQUISA}

Não se aplica

\section{FINANCIAMENTO}

Não se aplica.

\section{AGRADECIMENTO}

Não se aplica.

\section{REFERÊNCIAS}

1. Ministério da Saúde (BR). Resolução do Conselho Nacional da Saúde n. 573, de 31 de janeiro de 2018. Recomendações do Conselho Nacional de Saúde à proposta de Diretrizes Curriculares Nacionais do curso de graduação Bacharelado em Enfermagem. Diário Oficial da União [periódico na internet], Brasília (DF). 06 nov 2018 [citado 10 set 2021]. Disponível em: https://www.in.gov.br/materia/-/asset_publisher/Kujrw0TZC2Mb/content/id/48743098/do1-2018-11-06-resolucao-n-573-de31-de-janeiro-de-2018-48742847

2. Santos VLCG, Cesaretti IUR. Assistência em Estomaterapia: Cuidando de Pessoas com Estomia. $2^{\circ}$ ed. São Paulo: Atheneu; 2015.

3. World Council of Enterostomal Therapists (WCET). Mission, Values \& Vision [Internet]. [Acesso em 10 set 2021 ]. Disponível em: www.wcetn.org

4. Gontijo TG, Borges EL, Ferraz AF, Pires Júnior JF, Spira JAO. Atuação profissional dos estomaterapeutas egressos da Universidade Federal de Minas Gerais. ESTIMA Braz J Enterostomal Ther. 2019;17:e1419. https://doi.org/10.30886/estima.v17.686_PT

5. Gonçalves FGA, Reis FLM, Silva NAB, Souza NVDO, Varella TCMML, Pires AS. Conteúdo de estomaterapia e estratégias de ensino no currículo de graduação em enfermagem. Rev. Enferm UERJ. 2018;26:e28921. http://doi.org/10.12957/reuerj.2018.28921

6. Rodrigues RA, Bueno AA, Silva LM, Kusumota L, Almeida VC, Giacomini SB et al. O ensino de enfermagem gerontológica nas instituições públicas brasileiras de ensino superior. Acta Paul Enferm. 2018;31(3):313-20. https://doi.org/10.1590/19820194201800044

7. Oliveira DC. Análise de conteúdo temático-categorial: uma proposta de sistematização. Rev. enferm. UER]. 2008; [acesso em 10 set 2021];16(4):569-76. Disponível em: https://pesquisa.bvsalud.org/portal/resource/pt/bde-16162

8. Conselho Federal de Enfermagem, Brasília. Resolução Cofen n. 0567/2018. Regulamento da atuação da equipe de enfermagem no cuidado aos pacientes com feridas. Disponível em: http://www.cofen.gov.br/wp-content/uploads/2018/02/ANEXORESOLU\%C3\%87\%C3\%830-567-2018.pdf

9. SilvaJLG, Oliveira-Kumakura ARS. Clinical simulation to teach nursing care for wounded patients. Rev Bras Enferm. 2018;71(Suppl 4):1785-90. http://doi.org/10.1590/0034-7167-2017-0170

10. Alves NP, Gomes TG, Lopes MMCO, Gubert FA, Lima MA, Beserra EP et al. Simulação realística e seus atributos para a formação do enfermeiro. Rev enferm UFPE online. 2019; [acesso em 09 set 2021];13(5):1420-8. Disponível em: https://periodicos.ufpe. $\mathrm{br} /$ revistas/revistaenfermagem/article/view/239014/32337

11. Silva KCS, Barros MBSC, Silva VAO, Sanches LMP, Neto JAM, Alves SJO. Gamificação como Tecnologia Educacional Auxiliadora no Ensino de Curativos. In: Congresso sobre tecnologias na educação. 2019;504-10. https://doi.org/10.5753/ctrle.2019.8923 
12. Marques BLC, Chave USB, Costa CCP. Relato de experiência sobre a criação de um jogo educativo acerca dos conteúdos de estomaterapia para estudantes do curso de enfermagem. Revista Presença. 2019; [acesso em 10 set 2021];1(13). Disponível em: http://sistema.celsolisboa.edu.br/ojs/index.php/numerohum/article/view/198/144

13. Agência Nacional de Vigilância Sanitária (BR). Núcleos de Segurança do Paciente. Relatórios Eventos Adversos dos Estados. Incidentes relacionados à assistência à saúde. Resultados das notificações realizadas no Notivisa - Brasil, junho de 2019 a maio de 2020 [acesso em 10 set 2021]. Disponível em: https://www20.anvisa.gov.br/segurancadopaciente/index.php/publicacoes/ category/relatorios-dos-estados

14. Moreno-Monsiváis MG. Practice Based on Scientific Evidence as the Foundation of Clinical Practice. Aquichan. 2019;19(3):e1931. https://doi.org/10.5294/aqui.2019.19.3.1

15. Caliri MHL, Santos VLCG, Mandelbaum MHS, Costa MSN IG. Classificação das lesões por pressão - Consenso NPUAP 2016 Adaptada culturalmente para o Brasil. [internet] Associação Brasileira de Estomaterapia SOBEST e Associação Brasileira de Enfermagem em Dermatologia- SOBENDE [acesso em 10 set 2021]. Disponível em: http://www.sobest.org.br/textod/35

16. Yamada B. Associação Brasileira de Estomaterapia: estomias, feridas e incontinências - SOBEST [Internet]. Fundação. 2016 [acesso em 10 set 2021]. Disponível em: http://www.sobest.org.br/texto/3

17. Souza NVDO, David HMSL, Mauricio VC, Costa CCP. Enfermagem em Estomaterapia no telessaúde UERJ: Relato de Experiência. J Bras Tele. 2014;3(2):70-2. https://doi.org/10.12957/jbrastele.2014.13593

18. Baratieri T, Sangaleti CT, Trincaus MR. Conhecimento de acadêmicos de enfermagem sobre avaliação e tratamento de feridas. Rev Enferm Atenção Saúde. 2015; [acesso em 10 set 2021];4(1):2-15. Disponível em: http://seer.uftm.edu.br/revistaeletronica/ index.php/enfer/article/view/1259

19. Furtado $A F$, Marcondes L, Lenhani BE, Batista J. Conhecimento de acadêmicos de enfermagem sobre lesões por pressão: desafio para a segurança do paciente. Rev baiana enferm. 2019;33:e34425. https://doi.org/10.18471/rbe.v33.34425

20. Luo Y, Parry M, Huang YJ, Wang XH, He GP. Nursing students' Knowledge and atitudes toward urinary incontinence: A cross sectional survey. Nurse Educ Today. 2016;40:134-9. https://doi.org/10.1016/j.nedt.2016.02.020

21. Zimnicki K, Pieper B. Assessment of prelicensure undergraduate baccalaureate nursing students: ostomy knowledge, skill experiences, and confidence in care. Ostomy Wound Manage. 2018;64(8):35-42. https://doi.org/10.25270/owm.2018.8.3542

22. Ministério da Educação (BR). Resolução n. 7 Conselho Nacional de Educação, de 18 de dezembro de 2018. Estabelece as Diretrizes para a Extensão na Educação Superior Brasileira. Diário Oficial da União [periódico na internet], Brasília (DF). 19 dez 2018 [citado 10 set 2021]. Disponível em: http://portal.mec.gov.br/index.php?option=com_docman\&view=download\&ali as=104251-rces007-18\&category_slug=dezembro-2018-pdf\&Itemid=30192

23. Carvalho NAR, Nolêto IRSG, Santos JDMS, Benício CDAV, Bezerra SMG, Luz MHBA. Vivências de acadêmicos de enfermagem em uma liga de estomaterapia. Rev Enferm UFPI. 2015;4(4):105-8. https://doi.org/10.26694/reufpi.v4i4.3045

24. Prado ARA, Barreto VPM, Tonini T, Silva AS, Machado WCA. O saber do enfermeiro na indicação de coberturas no cuidado ao cliente com feridas. ESTIMA Braz J Enterostomal Ther. 2016;14(4):175-82. https://doi.org/10.5327/Z1806-3144201600040004 